\title{
Flux form Semi-Lagrangian methods for parabolic problems
}

\author{
Luca Bonaventura $^{1^{*}}$, Roberto Ferretti $^{2}$ \\ ${ }^{1} \mathrm{MOX}$ - Modelling and Scientific Computing, Dipartimento di Matematica \\ Politecnico di Milano, Milan, Italy \\ ${ }^{2}$ Dipartimento di Matematica e Fisica, \\ Università degli Studi Roma Tre, Rome, Italy \\ *Email address for correspondence: luca.bonaventura@polimi.it \\ Communicated by Maurizio Falcone \\ Received on May 29, 2015. Accepted on March 20, 2016.
}

\begin{abstract}
A semi-Lagrangian method for parabolic problems is proposed, that extends previous work by the authors to achieve a fully conservative, flux-form discretization of linear and nonlinear diffusion equations. A basic consistency and stability analysis is proposed. Numerical examples validate the proposed method and display its potential for consistent semi-Lagrangian discretization of advection-diffusion and nonlinear parabolic problems.

Keywords: Semi-Lagrangian methods, Flux-Form Semi-Lagrangian methods, Diffusion equations, Divergence form.

AMS subject classification: 35K10, 35L02, 65M08, 65M25.
\end{abstract}

\section{Introduction}

Semi-Lagrangian (SL) schemes were developed, in their original formulation, for the case of purely hyperbolic equations and do not guarantee conservation of mass. In recent years, an increasing number of SL discretization approaches have been proposed to circumvent these limitations.

While the "classical" technique is to treat in Eulerian form diffusion terms within SL models, various generalizations of the SL strategy to second order, parabolic problems have been proposed over the last two decades. We will refer here to selected works, while a more comprehensive review can be found in [1]. A first example of SL method for parabolic problems was proposed in [2], while fully probabilistic approaches were proposed in [3], [4], [5]. Deterministic discretizations inspired by stochastic considerations have been presented in [6] and [7].

In spite of the variety of applications involved, the common feature of these works is to replace the computation of the solution at the foot of a characteristic with a weighted average of values computed at multiple points. A rigorous derivation of these techniques can be based on the Feynman-Kač representation formula, with stochastic trajectories integrated backward in time as in conventional SL schemes. However, due to their stochastic origin, all these generalizations of the SL approach are based on the trace form of 


\section{Bonaventura, Ferretti}

parabolic problems, and unsuitable for the divergence form

$$
u_{t}=\operatorname{div}(D \nabla u),
$$

which is the most widely used in many applications, especially in computational fluid dynamics on geophysical scales, for which SL methods for advective problems have proven to be especially useful (see e.g. the review in [8]). In the previous paper [9], we have proposed for the first time a SL method for parabolic problems in divergence form. Numerical experiments reported therein show that the method, albeit formally only firstorder accurate in time, allows to compute remarkably accurate approximations of linear and nonlinear parabolic problems, as well as to achieve easily higher order accuracy in space also on nonuniform meshes.

On the other hand, the last two decades have witnessed an increasing interest in conservative, flux-form SL (FFSL) schemes for various linear and nonlinear advective problems. Some of the earliest and best known techniques of this kind were proposed in a finite volume framework in [10], [11] for applications to numerical weather prediction and climate modelling. Similar ideas have also been proposed in [12], [13], [14], for applications to plasma modelling and flow in porous media, while extensions of this ideas to the Discontinuous Galerkin framework have been first proposed in [15] and later also in [16], [17], [18]. All these methods are exactly mass conservative, as opposed to methods based on upstream remapping of computational cells (also called inherently conservative in the numerical weather prediction literature). Some early examples of the latter are to be found in [19], [20], [21], [22], [23]. Extensions to spherical geometry were proposed in [24], [25], [26]. An important representative of this class of techniques is the so called SLICE method, proposed and analyzed in a series of papers by Met Office researchers [27], [28], [29], [30].

The method proposed in [9], however, even though discretizing Equation (1) directly, is not formulated in flux form at the discrete level and does not guarantee exact mass conservation. In the present work, we try to fill the gap, by extending the approach of [9] to achieve a fully conservative, flux form discretization of the diffusion equation (1). Along the same lines as [9], we outline a consistency and stability analysis of the method in a simplified setting, and perform several numerical simulations to validate the proposed method.

The paper is structured as follows. In Section 2, our novel FFSL discretization is introduced, while an analysis of its consistency and stability properties will be presented in Sections 3 and 4. Section 5 treats the extensions of the scheme to problems in higher dimensions. Finally, numerical results obtained with the proposed method for both linear and nonlinear models will be presented in Section 6, while some conclusions on the potential advantages of our approach will be drawn in Section 7 .

\section{Flux Form Semi-Lagrangian methods for second order prob- lems}

In order to sketch the basic ideas of the scheme, we restrict for the moment to the approximation of the purely diffusive equation

$$
u_{t}=\left(\nu(x, t) u_{x}\right)_{x}
$$

in a single space dimension, considered for simplicity on the whole real line. The extension to advection-diffusion equations and to multiple dimensions will be discussed later in this Section and in Section 5. Extension to bounded domains with flux conditions at the 


\section{Flux form SL methods for parabolic problems}

boundary can be performed by a suitable modification of the numerical fluxes, following the same steps that would be taken in a standard finite volume scheme.

Let $\Delta t$ and $\Delta x$ denote the time and space discretization steps, respectively, with $t_{n}=n \Delta t$ for $n \in[0, T / \Delta t]$. The space grid is supposed to be infinite and uniform, that is, for $i \in \mathbb{Z}, x_{i}=i \Delta x$. We will also consider the intermediate points $x_{i \pm 1 / 2}=(i \pm 1 / 2) \Delta x$, which will appear as endpoints of grid cells $\Omega_{i}=\left[x_{i-1 / 2}, x_{i+1 / 2}\right]$. We will denote the numerical solutions at time $t_{n}$ by the vector $V^{n}=\left(v_{i}^{n}\right)_{i \in \mathbb{Z}}$. While in the nonconservative form SL scheme these values should be understood as point values, in a FFSL scheme they must be interpreted as cell averages.

We first provide a heuristic derivation of the proposed scheme, describing later in detail its link to the non conservative method we proposed in [9]. A finite volume, flux form approximation of Equation (2) can be written as a discrete mass balance

$$
\bar{u}_{i}\left(t_{n+1}\right) \approx \bar{u}_{i}\left(t_{n}\right)+\frac{1}{\Delta x}\left(\mathcal{F}_{i+1 / 2}^{n}-\mathcal{F}_{i-1 / 2}^{n}\right),
$$

where the cell averages are defined as

$$
\bar{u}_{i}(t) \approx \frac{1}{\Delta x} \int_{\Omega_{i}} u(x, t) d x .
$$

Denoting by $\delta=\sqrt{2 \Delta t \nu}$ the spatial scale naturally associated to the given diffusion over a time step $\Delta t$, one can write

$$
\begin{aligned}
\mathcal{F}_{k}^{n} & \approx \Delta t \nu\left(x_{k}, t_{n}\right) u_{x}\left(x_{k}, t_{n}\right) \approx \frac{\delta^{2}}{2} u_{x}\left(x_{k}, t_{n}\right) \approx \\
& \approx \frac{\delta}{2}\left(u\left(x_{k}+\frac{\delta}{2}, t_{n}\right)-u\left(x_{k}-\frac{\delta}{2}, t_{n}\right)\right),
\end{aligned}
$$

where the derivative has been approximated by a centered finite difference. Moreover, it can be observed that, applying the midpoint quadrature rule, one has

$$
\begin{aligned}
& \delta u\left(x_{k}+\frac{\delta}{2}, t_{n}\right) \approx \int_{x_{k}}^{x_{k}+\delta} u\left(x, t_{n}\right) d x, \\
& \delta u\left(x_{k}-\frac{\delta}{2}, t_{n}\right) \approx \int_{x_{k}-\delta}^{x_{k}} u\left(x, t_{n}\right) d x,
\end{aligned}
$$

so that one could define a mass flux as

$$
\mathcal{F}_{k}^{n}=\frac{1}{2}\left(\int_{x_{k}}^{x_{k}+\delta} u\left(x, t_{n}\right) d x-\int_{x_{k}-\delta}^{x_{k}} u\left(x, t_{n}\right) d x\right) .
$$

This definition is consistent with the physical interpretation of the diffusion process. Indeed, Equation (2) is based implicitly on Fick's law, according to which the effect of diffusion is to move mass from regions with high density towards regions with low density. The difference of integrals in (4) can interpreted as an approximation of the diffusive mass balance. This motivates the definition of the flux $\mathcal{F}_{k}^{n}$ in abstract form as

$$
\mathcal{F}_{k}^{n}=\frac{1}{2}\left(\int_{x_{k}}^{x_{k}+\delta_{k}} u\left(x, t_{n}\right) d x-\int_{x_{k}-\delta_{k}}^{x_{k}} u\left(x, t_{n}\right) d x\right)
$$

for a properly defined $\delta_{k}$.

The practical version of the scheme needs a further discretization in space for the (approximate) computation of the integrals. In the space-discrete framework, the flux (5) 


\section{Bonaventura, Ferretti}

is implemented with a numerically reconstructed function, so that the proposed FFSL method for Equation (2) is defined as

$$
v_{i}^{n+1}=v_{i}^{n}+\frac{1}{\Delta x}\left(F_{i+1 / 2}^{n}-F_{i-1 / 2}^{n}\right)
$$

with a numerical flux given, for $k=i \pm 1 / 2$, by

$$
F_{k}^{n}=\frac{1}{2}\left(\int_{x_{k}}^{x_{k}+\delta_{k}} R_{q}\left[V^{n}\right](x) d x-\int_{x_{k}-\delta_{k}}^{x_{k}} R_{q}\left[V^{n}\right](x) d x\right),
$$

and with

$$
\delta_{k}=\sqrt{2 \Delta t \nu\left(x_{k}, t_{n}\right)}
$$

Here, given a sequence $W=\left\{w_{m}\right\}_{m \in \mathbb{Z}}$ of cell averages, the operator $R_{q}[W]$ is a polynomial reconstruction of degree $q$ which satisfies over each cell $\Omega_{m}=\left[x_{m-1 / 2}, x_{m+1 / 2}\right]$ the properties of having the required mean value

$$
\frac{1}{\Delta x} \int_{\Omega_{m}} R_{q}[W] d x=w_{m}
$$

and reconstructing with high order a smooth solution. We refer to [31] for an in-depth discussion of these theoretical issues, while in Sections 3-4 we will recall the main points of interest for the purpose of a consistency and stability analysis of the proposed method.

It should be remarked that, in this case, it is not required to solve any equation to obtain $\delta_{k}$, in contrast to the approach that was necessary for the non conservative version of [9]. Furthermore, notice that the method (6) (as well as the nonconservative version, which will be recalled later) employs viscosity values frozen at the time level $t_{n}$, so that an extension to the nonlinear case is straightforward. Consistency (and its optimal rate) will be proven in the next Section.

The proposed FFSL method can also be combined with analogous methods for the flux form advection equation. Consider the advection-diffusion equation

$$
u_{t}+(f(x, t) u)_{x}=\left(\nu(x, t) u_{x}\right)_{x},
$$

taken again for simplicity on the infinite real line. The combination of the FFSL methods for advection and diffusion is obtained, for the purposes of this paper, by a simple operator splitting approach. This combination results in a method that is first order accurate in time, which is compatible with the time accuracy of the FFSL method for diffusion, as it will be seen in Section 3. In a first step, any FFSL method for advection can be used. In Section 6, we employ for concreteness a variant of the well-known FFSL method presented in [11]. This method yields a numerical approximation $\tilde{v}_{i}^{n+1}$ of the cell averages of the solution of (9) with $\nu=0$. An approximation of the solution of the complete Equation (9) is then obtained by computation of formula (6) based on the intermediate values $\tilde{v}_{i}^{n+1}$.

\section{Consistency}

We have shown in [9] that (2) can be approximated via an abstract difference operator in the form of a convex combination of point values. The results of this approach are closely related to the FFSL scheme under consideration, so we recall them here.

In [9], the convex combination takes the specific, nonconservative form

$$
v_{i}^{n+1}=\frac{1}{2} I_{p}\left[V^{n}\right]\left(x_{i}+\delta_{i}^{+}\right)+\frac{1}{2} I_{p}\left[V^{n}\right]\left(x_{i}-\delta_{i}^{-}\right),
$$




\section{Flux form SL methods for parabolic problems}

where the point values are reconstructed by the interpolation operator (of degree $p$ ) $I_{p}\left[V^{n}\right]$, while the displacements $\delta_{i}^{ \pm}$are given as solution of

$$
\delta_{i}^{ \pm}=\sqrt{2 \Delta t \nu\left(x_{i} \pm \delta_{i}^{ \pm}, t_{n}\right)}
$$

Notice that, in general, the interpolation operator $I_{p}$ differs from the polynomial reconstructions $R_{q}[W]$ introduced in Section 2, in that $I_{p}$ employs as input data point values, while $R_{q}[W]$ employs cell averages and provides a reconstruction that preserves cell averages exactly. To fix ideas, we assume that $I_{p}$ is in the form of a symmetric Lagrange interpolation, for odd values of $p$. For example, if $p=3$, the value $I_{3}[V](x)$ is computed, for $x \in\left[x_{m}, x_{m+1}\right]$, by interpolating the values $v_{m-1}, \ldots, v_{m+2}$. Equation (11) can be solved either by bisection or (if $\nu$ is uniformly positive and Lipschitz continuous) by fixed point iterations. In the case of a constant coefficient equation, it reduces to

$$
\delta=\sqrt{2 \Delta t \nu},
$$

i.e., the usual definition of $\delta$ used in schemes motivated by probabilistic considerations. It turns out (see [9]) that the consistency error of the scheme (10) has the form:

$$
L(\Delta x, \Delta t)=O\left(\Delta t+\frac{\Delta x^{p+1}}{\Delta t}\right) .
$$

To prove a sharp consistency estimate for the flux form SL method (6), we will work on cell averages, assuming as usual that both $\nu$ and $u$ are smooth functions of $x$ and $t$, and that the grid is uniform and infinite. First, integrating (2) on the generic cell $\Omega_{i}$, we get

$$
\int_{\Omega_{i}} u_{t} d x=\nu\left(x_{i+1 / 2}, t\right) u_{x}\left(x_{i+1 / 2}, t\right)-\nu\left(x_{i-1 / 2}, t\right) u_{x}\left(x_{i-1 / 2}, t\right) .
$$

Dividing now by $\Delta x$ to obtain the cell average, and using a first order Taylor expansion in time, we have

$$
\begin{aligned}
\bar{u}_{i}\left(t_{n+1}\right)= & \bar{u}_{i}\left(t_{n}\right)+\frac{\Delta t}{\Delta x}\left(\nu\left(x_{i+1 / 2}, t_{n}\right) u_{x}\left(x_{i+1 / 2}, t_{n}\right)-\right. \\
& \left.-\nu\left(x_{i-1 / 2}, t_{n}\right) u_{x}\left(x_{i-1 / 2}, t_{n}\right)\right)+O\left(\Delta t^{2}\right) .
\end{aligned}
$$

On the other hand, we can rewrite more precisely the abstract, time-discrete approximation (3) as

$$
\bar{u}_{i}\left(t_{n+1}\right)=\bar{u}_{i}\left(t_{n}\right)+\frac{1}{\Delta x}\left(\mathcal{F}_{i+1 / 2}^{n}-\mathcal{F}_{i-1 / 2}^{n}\right)+O\left(\Delta t^{r+1}\right),
$$

with $r$ the associated consistency rate. Denoting by $U(x, t)$ the primitive function (with respect to $x)$ of the solution $u(x, t)$, we can recast the abstract flux $\mathcal{F}_{k}^{n}$ as

$$
\begin{aligned}
\mathcal{F}_{k}^{n} & =\frac{1}{2}\left(U\left(x_{k}+\delta_{k}, t_{n}\right)-2 U\left(x_{k}, t_{n}\right)+U\left(x_{k}-\delta_{k}, t_{n}\right)\right)= \\
& =\frac{\delta_{k}^{2}}{2} U_{x x}\left(x_{k}, t_{n}\right)+\frac{\delta_{k}^{4}}{24} U_{x x x x}\left(x_{k}, t_{n}\right)+O\left(\delta_{k}^{6}\right)= \\
& =\Delta t \nu\left(x_{k}, t_{n}\right) u_{x}\left(x_{k}, t_{n}\right)+\frac{\Delta t^{2} \nu^{2}\left(x_{k}, t_{n}\right)}{6} u_{x x x}\left(x_{k}, t_{n}\right)+O\left(\Delta t^{3}\right) .
\end{aligned}
$$

Using (14) in the right-hand side of (13) (for $k=i \pm 1 / 2$ ), we get

(15) $\bar{u}_{i}\left(t_{n}\right)+\frac{1}{\Delta x}\left(\mathcal{F}_{i+1 / 2}^{n}-\mathcal{F}_{i-1 / 2}^{n}\right)=\bar{u}_{i}\left(t_{n}\right)+\frac{\Delta t}{\Delta x}\left(\nu\left(x_{i+1 / 2}, t_{n}\right) u_{x}\left(x_{i+1 / 2}, t_{n}\right)-\right.$ $\left.-\nu\left(x_{i-1 / 2}, t_{n}\right) u_{x}\left(x_{i-1 / 2}, t_{n}\right)\right)+O\left(\Delta t^{2}\right)$. 


\section{Bonaventura, Ferretti}

In deriving (15), we have taken into account that

$$
\frac{\Delta t^{2} \nu^{2}\left(x_{i+1 / 2}, t_{n}\right)}{6} u_{x x x}\left(x_{i+1 / 2}, t_{n}\right)-\frac{\Delta t^{2} \nu^{2}\left(x_{i-1 / 2}, t_{n}\right)}{6} u_{x x x}\left(x_{i-1 / 2}, t_{n}\right)=O\left(\Delta t^{2} \Delta x\right)
$$

due to the Lipschitz continuity of $\nu$ and $u_{x x x}$. Comparing now (15) with (12), we see that (13) is satisfied up to an $O\left(\Delta t^{2}\right)$, which means a consistency of order $r=1$ with respect to $\Delta t$.

In the second discretization step, we introduce the polynomial reconstruction $R_{q}[V]$ to compute the fluxes in the numerical form (7). Then, the integrals in (7) are computed with some given accuracy depending (only) on $\Delta x$, so that adding this extra error term we get a final consistency error $L(\Delta x, \Delta t)$ of the form

$$
L(\Delta x, \Delta t)=O\left(\Delta t+\frac{\Delta x^{s}}{\Delta t}\right)
$$

The exponent $s$ in (16) depends on the kind of space reconstruction used. A particular choice which provides a stable scheme is obtained by defining the reconstruction $R_{q}$ (see [31] for more details and examples) according to the axioms

i) For $x \in \Omega_{m}, R_{q}[W](x)=Q_{m}(x)$ for some polynomial $Q_{m} \in \mathbb{P}_{q}$, with $q$ even;

ii) For $x \in \Omega_{m}, Q_{m}(x)$ depends on the values $w_{k}$, with $k=m-q / 2, \ldots, m+q / 2$, and more precisely it satisfies the conditions

$$
\frac{1}{\Delta x} \int_{\Omega_{k}} Q_{m}(x) d x=w_{k} \quad(k=m-q / 2, \ldots, m+q / 2) .
$$

With this definition, it is proven in [31] that

$$
\frac{1}{\Delta x} \int_{x-\Delta x / 2}^{x+\Delta x / 2} R_{q}[W](\xi) d \xi=I_{p}[W](x),
$$

for $p=q+1$ and $I_{p}$ in the form of a symmetric Lagrange interpolation.

Equation (17) shows that the integral average over a cell (or part of a cell) is computed with accuracy $O\left(\Delta x^{q+2}\right)$ for a smooth solution. To evaluate the effect of numerical reconstruction on the computation of the integrals in (7), note that the domains of integration in the definition of the fluxes $F_{k}^{n}$ are $\left[x_{k}, x_{k}+\delta_{k}\right]$ or $\left[x_{k}-\delta_{k}, x_{k}\right]$. In both cases, the contribution to the integral of any cells $\Omega_{j}$ which is fully included in the integration interval is exactly computed as $\Delta x v_{j}^{n}$, and numerical errors in the computation of the fluxes $F_{k}^{n}$ are associated to at most one incomplete cell in each integration domain. Therefore,

$$
\frac{1}{\Delta x}\left(\mathcal{F}_{k}^{n}-F_{k}^{n}\right)=O\left(\Delta x^{q+2}\right),
$$

and the estimate (16) holds for the truncation error $L$, with $s=q+2$.

\section{Stability}

We briefly discuss the stability of the proposed scheme in the case of a constant coefficients equation, while variable coefficient equations seem to require a deeper study. First, we note that a well understood case is the construction of the nonconservative scheme with $I_{p}$ in the form of a symmetric Lagrange interpolation, for odd values of $p$. In the pure advection case, this construction is known to be stable (see [32]). 


\section{Flux form SL methods for parabolic problems}

Consider now the problem (2) for a constant diffusivity $\nu$, and define the space reconstruction $R_{q}$ as in the previous section. In this case, $\delta_{i} \equiv \delta$, so that, writing explicitly the fully discrete scheme and using (17), we get

$$
\begin{aligned}
v_{i}^{n+1} & =v_{i}^{n}+\frac{1}{\Delta x}\left(F_{i+1 / 2}^{n}-F_{i-1 / 2}^{n}\right)= \\
(18) & =v_{i}^{n}+\frac{1}{2 \Delta x}\left(\int_{x_{i-1 / 2}-\delta}^{x_{i+1 / 2}-\delta}-\int_{x_{i-1 / 2}}^{x_{i+1 / 2}}+\int_{x_{i-1 / 2}+\delta}^{x_{i+1 / 2}+\delta}-\int_{x_{i-1 / 2}}^{x_{i+1 / 2}}\right) R_{q}\left[V^{n}\right](x) d x= \\
& =\frac{1}{2}\left(\frac{1}{\Delta x} \int_{x_{i-1 / 2}-\delta}^{x_{i+1 / 2}-\delta}+\frac{1}{\Delta x} \int_{x_{i-1 / 2}+\delta}^{x_{i+1 / 2}+\delta}\right) R_{q}\left[V^{n}\right](x) d x= \\
& =\frac{1}{2} I_{p}\left[V^{n}\right](x-\delta)+\frac{1}{2} I_{p}\left[V^{n}\right](x+\delta) .
\end{aligned}
$$

The scheme can then be recast as the convex combination

$$
V^{n+1}=\frac{1}{2} B^{+} V^{n}+\frac{1}{2} B^{-} V^{n},
$$

where the terms $B^{ \pm} V^{n}$ represent respectively the schemes written in extended form as

$$
v_{i}^{n+1}=I_{p}\left[V^{n}\right]\left(x_{i} \pm \delta\right),
$$

which (see [32]) are stable in the 2-norm for any $\delta$ and any odd $p$. This implies that

$$
\left\|B^{ \pm}\right\|_{2} \leq 1
$$

and therefore that the complete scheme is also stable in the same norm, unconditionally with respect to $\Delta x$ and $\Delta t$.

This unconditional stability, however, comes at a price. The numerical domain of dependence, as (19) shows, is made of two separate regions at a distance of about $2 \delta$ (or $2 \delta_{k}$ for variable coefficient problems). In case of a strong diffusivity, the use of large time steps may cause the small scales to be severely underresolved, especially in the first steps of the scheme and for nonsmooth initial conditions, although the scheme remains formally stable and consistent.

A qualitative evaluation of this phenomenon is carried out in [6]. The endpoint of this analysis is a compatibility condition of the form

$$
\frac{\|\nu\|_{\infty}^{1 / 3} \Delta t}{T^{2 / 3} \Delta x^{2 / 3}} \ll 1
$$

in which $T$ is the time of interest for the approximation of the solution. Note that this condition is less restrictive for large $T$ and small $\nu$, and is asymptotically satisfied under hyperbolic-type CFL conditions (in particular, it is satisfied for all the space-time grids of Section 6). An adaptive strategy of selection of the time step has also been proposed in [33] for a similar scheme related to the equation of Mean Curvature Motion.

\section{Multiple space dimensions}

In this Section, we discuss the extension of the proposed approach to the $d$ dimensional case. The extension is straightforward in the case of a structured orthogonal grid and diagonal diffusivity matrix

$$
\Lambda(x, t)=\operatorname{diag}\left(\nu_{1}(x, t), \ldots, \nu_{d}(x, t)\right),
$$




\section{Bonaventura, Ferretti}

and in particular, for a variable but isotropic diffusion (for which $\nu_{1}(x, t)=\cdots=\nu_{d}(x, t)$ ). Then, the diffusion equation reads

$$
\begin{aligned}
u_{t} & =\operatorname{div}(\Lambda(x, t) \nabla u)= \\
& =\sum_{j=1}^{d}\left(\nu_{j}(x, t) u_{x_{j}}\right) x_{j} .
\end{aligned}
$$

By a first-order expansion of the solution with respect to time, we get

$$
u(x, t+\Delta t)=u(x, t)+\frac{1}{d} \sum_{j=1}^{d}\left[\Delta t\left(d \nu_{j} u_{x_{j}}\right)_{x_{j}}(x, t)\right]+O\left(\Delta t^{2}\right),
$$

which shows that, up to first-order accuracy, the $d$-dimensional version can be obtained by averaging the diffusion operators in each direction, with the only modification of scaling each one-dimensional diffusivity by a factor $d$. This allows to split the diffusion along each of the variables. For example, the 2-dimensional equation

$$
u_{t}=\left(\nu_{1}(x, t) u_{x_{1}}\right)_{x_{1}}+\left(\nu_{2}(x, t) u_{x_{2}}\right)_{x_{2}}
$$

would be approximated by the scheme (written with an obvious notation at the node $\left.x_{i}=\left(x_{i_{1}}, x_{i_{2}}\right)\right)$

$$
v_{i}^{n+1}=v_{i}^{n}+\frac{1}{\Delta x^{2}}\left(F_{i_{1}+1 / 2, i_{2}}^{n}-F_{i_{1}-1 / 2, i_{2}}^{n}+F_{i_{1}, i_{2}+1 / 2}^{n}-F_{i_{1}, i_{2}+1 / 2}^{n}\right),
$$

in which, for example, the flux $F_{i_{1}+1 / 2, i_{2}}^{n}$ would be given by

$$
\begin{aligned}
F_{i_{1}+1 / 2, i_{2}}^{n}= & \frac{1}{4}\left(\int_{x_{i_{2}-1 / 2}}^{x_{i_{2}+1 / 2}} \int_{x_{i_{1}+1 / 2}}^{x_{i_{1}+1 / 2}+\delta_{i_{1}+1 / 2, i_{2}}} R\left[V^{n}\right](x) d x-\right. \\
& \left.-\int_{x_{i_{2}-1 / 2}}^{x_{i_{2}+1 / 2}} \int_{x_{i_{1}+1 / 2}-\delta_{i_{1}+1 / 2, i_{2}}}^{x_{i_{1}+1 / 2}} R\left[V^{n}\right](x) d x\right),
\end{aligned}
$$

and the displacement $\delta_{i_{1}+1 / 2, i_{2}}$ defined, at the centers of $\mathrm{E}$ and $\mathrm{W}$ facets of a square cell, by

$$
\delta_{i_{1}+1 / 2, i_{2}}=\sqrt{4 \Delta t \nu_{1}\left(x_{i_{1}+1 / 2, i_{2}}, t_{n}\right)} .
$$

Note that the integrals appearing in all the fluxes of the form (22) have an integration domain consisting of a row (or column) of grid cells, among which at most one cell is incomplete (see the remark at the end of Section 3).

\section{Numerical experiments}

Several numerical experiments have been carried out with simple implementations of the FFSL method proposed above, in order to assess its accuracy and stability features also in cases more complex than those allowing a complete theoretical analysis. The accuracy of the proposed discretization has been evaluated against analytic solutions or reference solutions obtained by alternative discretizations in space and time. Due to the close relationship between the methods proposed here and those in [9], the choice of the test cases follows closely the outline in our previous paper, in order to allow for a clear comparison between the conservative and nonconservative SL discretizations. 


\section{Flux form SL methods for parabolic problems}

\subsection{Constant coefficient case}

In a first set of numerical experiments, the constant coefficient diffusion equation

$$
u_{t}=\nu u_{x x} \quad x \in[0, L]
$$

was considered, on an interval $[0, L]$ with $L=20$. Periodic boundary conditions were assumed and a Gaussian profile centered at $L / 2$ was considered as the initial condition. In this case, the exact solution can be computed up to machine accuracy by separation of variables and computation of its Fourier coefficients on a discrete mesh of $N$ points with spacing $\Delta x=L / N$. We consider the FFSL method described in the previous Sections on a time interval $[0, T]$ with $T=2$, with time steps defined as $\Delta t=T / M$. The stability parameter of standard explicit discretizations of the diffusion operator is defined as $\mu=$ $\nu \Delta t / \Delta x^{2}$. We consider the case with $\nu=0.05$ first, whose relative errors are reported in Table 1 , as computed in the $l_{2}$ norm $\left(l^{\infty}\right.$ norm errors have an entirely analogous behaviour). The parallel results for the advection diffusion case

$$
u_{t}+a u_{x}=\nu u_{x x} \quad x \in[0, L]
$$

with $a=1.5, \nu=0.05$ are reported in Table 2 (also in this case, $l^{\infty}$ norm errors are very similar). Here, the Courant number is defined as $C=a \Delta t / \Delta x$ and the advective flux was computed along the lines of the well known method [11], using the same $R_{q}$ reconstruction employed for the diffusive flux (and defined in Section 3), with $q=0$ and $q=2$, respectively.

Table 1. Relative errors for the constant coefficient pure diffusion case in the $l_{2}$-norm, nonconservative (SL) and conservative (FFSL) scheme, first and third order space discretizations.

\begin{tabular}{||c|c|c|c|c|c|c||}
\hline \multicolumn{3}{||c|}{ Resolution } & \multicolumn{2}{c|}{$l_{2}$ rel. error (SL) } & \multicolumn{2}{c||}{$l_{2}$ rel. error $(\mathrm{FFSL})$} \\
\hline$N$ & $M$ & $\mu$ & $I_{1}$ & $I_{3}$ & $R_{0}$ & $R_{2}$ \\
\hline \hline 400 & 12 & 3.3 & $1.50 \cdot 10^{-3}$ & $1.52 \cdot 10^{-4}$ & $1.56 \cdot 10^{-3}$ & $1.49 \cdot 10^{-4}$ \\
\hline 800 & 25 & 6.4 & $8.1 \cdot 10^{-4}$ & $7.14 \cdot 10^{-5}$ & $8.1 \cdot 10^{-4}$ & $7.1 \cdot 10^{-5}$ \\
\hline \hline 400 & 25 & 1.6 & $2.1 \cdot 10^{-3}$ & $8.02 \cdot 10^{-5}$ & $2.1 \cdot 10^{-3}$ & $7.21 \cdot 10^{-5}$ \\
\hline 800 & 50 & 3.2 & $1.56 \cdot 10^{-3}$ & $3.61 \cdot 10^{-5}$ & $1.56 \cdot 10^{-3}$ & $3.56 \cdot 10^{-5}$ \\
\hline \hline 400 & 50 & 0.8 & $4.78 \cdot 10^{-3}$ & $3.45 \cdot 10^{-5}$ & $4.78 \cdot 10^{-3}$ & $3.83 \cdot 10^{-5}$ \\
\hline 800 & 100 & 1.6 & $2.06 \cdot 10^{-3}$ & $1.72 \cdot 10^{-3}$ & $2.06 \cdot 10^{-3}$ & $1.80 \cdot 10^{-5}$ \\
\hline \hline 400 & 100 & 0.4 & $4.63 \cdot 10^{-3}$ & $4.17 \cdot 10^{-5}$ & $4.63 \cdot 10^{-3}$ & $2.04 \cdot 10^{-5}$ \\
\hline 800 & 200 & 0.8 & $4.76 \cdot 10^{-3}$ & $8.58 \cdot 10^{-6}$ & $4.76 \cdot 10^{-3}$ & $9.56 \cdot 10^{-6}$ \\
\hline \hline 400 & 200 & 0.2 & $2.21 \cdot 10^{-2}$ & $1.19 \cdot 10^{-4}$ & $2.21 \cdot 10^{-2}$ & $2.31 \cdot 10^{-5}$ \\
\hline 800 & 400 & 0.4 & $4.62 \cdot 10^{-3}$ & $1.1 \cdot 10^{-5}$ & $4.62 \cdot 10^{-3}$ & $5.09 \cdot 10^{-6}$ \\
\hline
\end{tabular}

According to the estimate (16), the consistency rate of the proposed method is at most one, due to predominance of time truncation errors. This behaviour is apparent when large values of the 'parabolic CFL parameter' $\mu$ are reached. In the opposite situation, when really many time steps are computed, the accumulation of space discretization errors kicks in, causing an increase of the second term in (16) and ultimately slowing down convergence - error tables thus confirm that the best performance of the scheme is achieved at intermediate Courant numbers. On the other hand, even at large Courant numbers the errors are comparable (or smaller, see the tests in the previous paper [9]) 


\section{Bonaventura, Ferretti}

Table 2. Relative errors for the constant coefficient advection-diffusion case in the $l_{2}$-norm, nonconservative (SL) and conservative (FFSL) scheme, first and third order space discretizations.

\begin{tabular}{||c|c|c|c|c|c|c|c||}
\hline \multicolumn{5}{|c|}{ Resolution } & \multicolumn{2}{c|}{$l_{2}$ rel. error $(\mathrm{SL})$} & \multicolumn{2}{c||}{$l_{2}$ rel. error $(\mathrm{FFSL})$} \\
\hline$N$ & $M$ & $\mu$ & $\mathrm{C}$ & $I_{1}$ & $I_{3}$ & $R_{0}$ & $R_{2}$ \\
\hline \hline 400 & 12 & 3.3 & 5 & $1.56 \cdot 10^{-3}$ & $1.52 \cdot 10^{-4}$ & $1.56 \cdot 10^{-3}$ & $1.50 \cdot 10^{-4}$ \\
\hline 800 & 25 & 6.4 & 4.8 & $6.88 \cdot 10^{-4}$ & $7.16 \cdot 10^{-5}$ & $1.3 \cdot 10^{-3}$ & $7.21 \cdot 10^{-5}$ \\
\hline \hline 400 & 25 & 1.6 & 2.4 & $2.45 \cdot 10^{-3}$ & $7.14 \cdot 10^{-5}$ & $5.03 \cdot 10^{-3}$ & $7.4 \cdot 10^{-5}$ \\
\hline 800 & 50 & 3.2 & 2.4 & $5.78 \cdot 10^{-4}$ & $3.7 \cdot 10^{-5}$ & $3.02 \cdot 10^{-3}$ & $4.24 \cdot 10^{-5}$ \\
\hline \hline 400 & 50 & 0.8 & 1.2 & $3.81 \cdot 10^{-3}$ & $4.27 \cdot 10^{-5}$ & $8.64 \cdot 10^{-3}$ & $4.07 \cdot 10^{-5}$ \\
\hline 800 & 100 & 1.6 & 1.2 & $1.57 \cdot 10^{-3}$ & $1.94 \cdot 10^{-5}$ & $4.0 \cdot 10^{-3}$ & $1.83 \cdot 10^{-5}$ \\
\hline \hline 400 & 100 & 0.4 & 0.6 & $1.11 \cdot 10^{-2}$ & $1.11 \cdot 10^{-4}$ & $1.61 \cdot 10^{-2}$ & $2.78 \cdot 10^{-5}$ \\
\hline 800 & 200 & 0.8 & 0.6 & $4.2 \cdot 10^{-3}$ & $1.43 \cdot 10^{-5}$ & $1.05 \cdot 10^{-2}$ & $1.06 \cdot 10^{-5}$ \\
\hline \hline 400 & 200 & 0.2 & 0.3 & $1.37 \cdot 10^{-2}$ & $3.41 \cdot 10^{-4}$ & $4.1 \cdot 10^{-2}$ & $3.59 \cdot 10^{-5}$ \\
\hline 800 & 400 & 0.4 & 0.3 & $9.65 \cdot 10^{-3}$ & $9.25 \cdot 10^{-5}$ & $1.47 \cdot 10^{-2}$ & $6.7 \cdot 10^{-6}$ \\
\hline
\end{tabular}

to those of unconditionally stable Eulerian schemes, but obtained at a much smaller computational cost, since no global linear algebraic problem is being solved here.

It can be observed that, in the linear case, the errors obtained by the SL and FFSL methods are in general of the same order of magnitude. Indeed, as a result of the analysis in [32], the SL and FFSL methods should give exactly identical results in the constant coefficient case, provided that the centered $I_{p}$ interpolation and the $R_{p-1}$ reconstructions are employed, respectively. The slight differences in Tables 1 and 2 are due to the fact that, for simplicity of the present implementation, for the SL method the default MATLAB cubic interpolator was used, which implements a shape preserving cubic interpolator that is equivalent in accuracy, but not exactly identical to the centered $I_{3}$ interpolator.

As expected, both methods have much smaller errors with cubic than with linear interpolation and the errors do not suffer from larger time step sizes, as typical with SL schemes, while it has been verified that mass conservation is respected up to machine accuracy.

\subsection{Linear diffusion with variable coefficients}

The diffusion equation

$$
u_{t}=\left(\nu(x, t) u_{x}\right)_{x} \quad x \in[0, L]
$$

was then considered on a space interval $[0, L]$ and time interval $[0, T]$ with $L=10$ and with $T=4$, with time steps defined as $\Delta t=T / M$. Periodic boundary conditions were assumed and a Gaussian profile centered at $L / 5$ was considered as the initial condition. The diffusivity field was given by

$$
\nu(x, t)=\frac{1}{20}+\frac{1}{5} \xi(x) \sin \left(\frac{2 \pi t}{T}\right)^{2},
$$

respectively, where $\xi(x)$ denotes the characteristic function of the interval $[0.5 L, 0.8 L]$. This choice highlights the possibility to use the proposed method seamlessly also with strongly varying diffusion coefficients. In this case, no exact solution is available and reference solutions were computed using the finite difference method described in the 


\section{Flux form SL methods for parabolic problems}

previous section with a four times higher spatial resolution, coupled to a high order multistep stiff solver in which a small tolerance and maximum time step value were enforced. A plot of the numerical solutions obtained at the final time $T$ are displayed in Figures 1-2, for the FFSL and SL method, respectively.

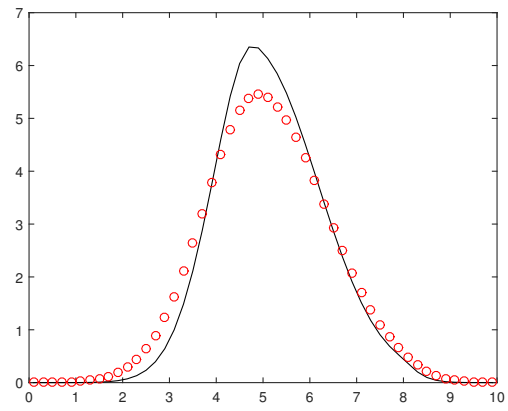

(a)

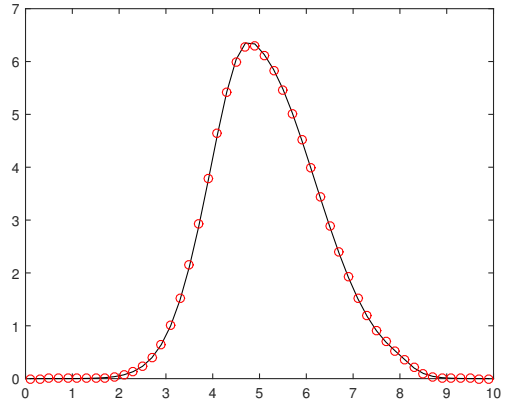

(b)

Figure 1. One-dimensional case with variable coefficients: reference solution (continuous line) versus numerical solution by FFSL method (circles) with first-order (a) and thirdorder (b) reconstruction.

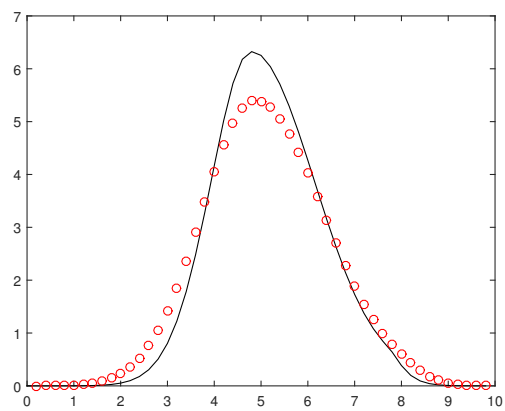

(a)

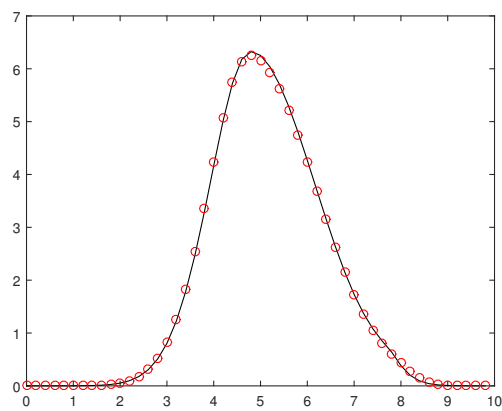

(b)

Figure 2. One-dimensional case with variable coefficients: reference solution (continuous line) versus numerical solution by SL method (circles) with linear (a) and cubic (b) interpolation.

A more quantitative assessment of the FFSL solution accuracy can be gathered from Table 3. It can be observed that the FFSL method has always slightly smaller error than the corresponding SL variant at equivalent resolution. In all these computations, FFSL maintains mass conservation up to machine accuracy by construction, while the average conservation error of the SL method is of the order of $10^{-3}$ times the total initial mass. 


\section{Bonaventura, Ferretti}

Table 3. Relative errors for the variable coefficient pure diffusion case in the $l_{2}$-norm, nonconservative (SL) and conservative (FFSL) scheme, first and third order space discretizations.

\begin{tabular}{||c|c|c|c|c|c|c||}
\hline \multicolumn{3}{||c|}{ Resolution } & \multicolumn{2}{c|}{$l_{2}$ rel. error (SL) } & \multicolumn{2}{c||}{$l_{2}$ rel. error (FFSL) } \\
\hline$N$ & $M$ & $\mu$ & $I_{1}$ & $I_{3}$ & $R_{0}$ & $R_{2}$ \\
\hline \hline 50 & 50 & 0.1 & $1.34 \cdot 10^{-1}$ & $1.10 \cdot 10^{-3}$ & $1.30 \cdot 10^{-1}$ & $7.31 \cdot 10^{-3}$ \\
\hline 100 & 100 & 0.2 & $7.26 \cdot 10^{-2}$ & $4.21 \cdot 10^{-3}$ & $7.21 \cdot 10^{-2}$ & $3.52 \cdot 10^{-3}$ \\
\hline \hline 100 & 25 & 0.8 & $2.10 \cdot 10^{-2}$ & $5.74 \cdot 10^{-3}$ & $2.10 \cdot 10^{-2}$ & $4.75 \cdot 10^{-3}$ \\
\hline 200 & 50 & 1.6 & $1.02 \cdot 10^{-2}$ & $4.68 \cdot 10^{-3}$ & $9.57 \cdot 10^{-3}$ & $3.00 \cdot 10^{-3}$ \\
\hline
\end{tabular}

\subsection{Gas flow in porous media}

We reconsider the nonlinear example, already treated in [9], of the one-dimensional equation of gases in porous media,

$$
u_{t}=\left(m u^{m-1} u_{x}\right)_{x}
$$

focusing in particular on the so-called Barenblatt-Pattle self-similar solutions (see, e.g., [34]), which can be written in the form

$$
u(x, t)=\left(t+t_{0}\right)^{-k}\left(A^{2}-\frac{k(m-1)|x|^{2}}{2 m\left(t+t_{0}\right)^{2 k}}\right)_{+}^{\frac{1}{m-1}}
$$

where $t_{0}>0, A$ is an arbitrary nonzero constant and $k=1 /(m+1)$.

In order to adapt the FFSL scheme to this case, we recall what has been already observed for the nonconservative scheme in [9]: since the solution (and hence, the diffusivity) may have a bounded support, a straightforward extension of (11) in the linearized form

$$
\delta_{i}^{ \pm}=\sqrt{2 \Delta t \nu\left(I\left[V^{n}\right]\left(x_{i} \pm \delta_{i}^{ \pm}\right)\right)}
$$

(where in our case $\nu(u)=m u^{m-1}$ ) might cause an improper behaviour of the scheme. In fact, for $x_{i}$ out of the support but in its neighbourhood, (25) could have multiple solutions, the smallest being $\delta_{i}^{ \pm}=0$. In order to ensure a correct propagation of the solution, $\delta_{i}^{ \pm}$should be selected to be the largest of such values, either starting the iterative computation from an initial guess exceeding all candidate solutions, or working by bisection.

A similar caution should be applied in extending the definition (8), which would only provide the value $\delta_{k}=0$ when outside of the support of the solution (note that this is not in contrast with the analysis of Section 3 which works for linear equations and under stronger assumptions). A possible answer in this respect is to compute the $\delta_{k}^{ \pm}$via (25), then define

$$
\delta_{k}=\frac{\delta_{k}^{+}+\delta_{k}^{-}}{2}
$$

where, of course, $x_{k}$ is now a cell interface. This means that in this case the FFSL scheme requires again to solve the scalar equation (25) at each node; however, as we will show, it turns out to be more accurate than the nonconservative scheme. 


\section{Flux form SL methods for parabolic problems}

We prove now that (26) is a consistent definition of $\delta_{k}$. By a Taylor expansion of the square root and some minor manipulation, we have

$$
\delta_{k}^{ \pm}=\sqrt{2 \Delta t \nu\left(x_{k}\right)}\left(1 \pm \frac{\nu_{x}\left(x_{k}\right)}{2 \nu\left(x_{k}\right)} \delta_{k}^{ \pm}+O(\Delta t),\right)
$$

so that

$$
\frac{\delta_{k}^{+}+\delta_{k}^{-}}{2}=\sqrt{2 \Delta t \nu\left(x_{k}\right)}\left(1+\frac{\nu_{x}\left(x_{k}\right)}{4 \nu\left(x_{k}\right)}\left(\delta_{k}^{+}-\delta_{k}^{-}\right)+O(\Delta t),\right) .
$$

On the other hand, it is proved in [9] that $\delta_{k}^{+}-\delta_{k}^{-}=O(\Delta t)$, and this implies that

$$
\frac{\delta_{k}^{+}+\delta_{k}^{-}}{2}=\sqrt{2 \Delta t \nu\left(x_{k}\right)}(1+O(\Delta t))
$$

i.e., that the definition (26), while allowing for nonzero values outside of the support of the solution, leaves the scheme consistent.

Figure 3 compares the exact and approximate evolution of the Barenblatt-Pattle solution for (23), with $m=3, A=1$ and $t_{0}=1$. Approximate solutions have been computed with the nonconservative (left) and the conservative (right) scheme at $T=1,4,16$ on a mesh composed of 50 nodes with $\Delta t=0.05$, using cubic interpolation and quadratic reconstruction, respectively. Note that the mass conservation constraint apparently improves the accuracy of the scheme, especially for larger simulation times.

This behaviour is confirmed, in terms of both relative error and convergence rate, by Table 4, which shows the numerical errors for the two schemes in the 2-norm, at $T=16$, under a linear refinement law. SL scheme has been tested with linear $\left(I_{1}\right)$ and cubic $\left(I_{3}\right)$ interpolation, whereas FFSL scheme with piecewise constant $\left(R_{0}\right)$ and piecewise quadratic $\left(R_{2}\right)$ reconstruction. When computed on the extreme discretizations of the table, the convergence rates are between 0.35 and 0.4 for the nonconservative scheme, and roughly doubled for the conservative scheme. Higher order space discretizations result in a similar convergence rate, but lower error.

The maximum values for the stability parameter $\mu$ are also displayed, showing that the scheme works well far beyond the time step prescribed according to a "parabolic CFL" condition.

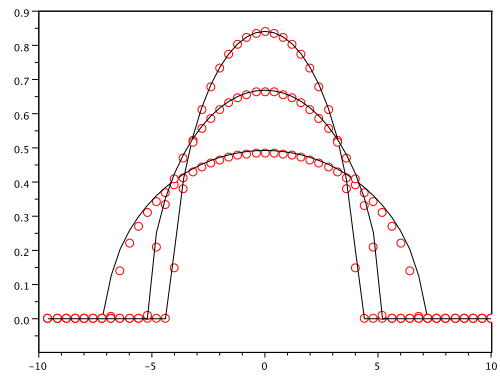

(a)

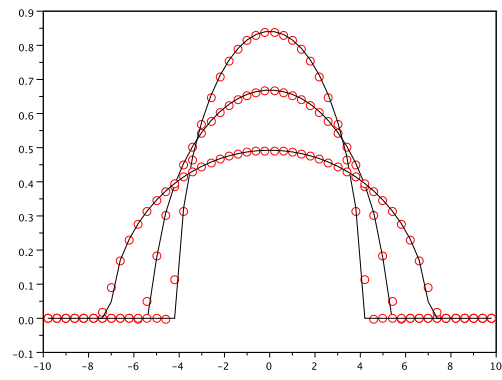

(b)

Figure 3. Evolution of the exact (continuous line) versus numerical (circles) BarenblattPattle solution for $T=1,4,16$, nonconservative (a) and conservative (b) scheme. 


\section{Bonaventura, Ferretti}

Table 4. Relative errors for the Barenblatt-Pattle solution in the 2-norm, nonconservative (SL) and conservative (FFSL) scheme, first and third order space discretizations.

\begin{tabular}{||c|c|c|c|c|c|c||}
\hline \multicolumn{3}{||c|}{ Resolution } & \multicolumn{2}{c|}{$l_{2}$ rel. error (SL) } & \multicolumn{2}{c||}{$l_{2}$ rel. error $(\mathrm{FFSL})$} \\
\hline$N$ & $M$ & $\mu$ & $I_{1}$ & $I_{3}$ & $R_{0}$ & $R_{2}$ \\
\hline 50 & 320 & 0.94 & 0.316 & $8.69 \cdot 10^{-2}$ & 0.270 & $2.67 \cdot 10^{-2}$ \\
\hline 100 & 640 & 1.9 & 0.212 & $4.76 \cdot 10^{-2}$ & 0.156 & $1.65 \cdot 10^{-2}$ \\
\hline 200 & 1280 & 3.8 & 0.171 & $4.84 \cdot 10^{-2}$ & $9.85 \cdot 10^{-2}$ & $1.09 \cdot 10^{-2}$ \\
\hline 400 & 2560 & 7.5 & 0.135 & $4.49 \cdot 10^{-2}$ & $6.03 \cdot 10^{-2}$ & $2.69 \cdot 10^{-3}$ \\
\hline 800 & 5120 & 15.0 & 0.107 & $3.22 \cdot 10^{-2}$ & $3.72 \cdot 10^{-2}$ & $2.79 \cdot 10^{-3}$ \\
\hline
\end{tabular}

\subsection{Variable coefficient case in two space dimensions}

As a two-dimensional test, we consider the equation

$$
u_{t}=\operatorname{div}(\nu(x) \nabla u)
$$

on $\Omega=[-3,3]^{2}$ with periodic boundary conditions. The initial condition given by the characteristic function of the square $\Sigma=[-1.5,1.5]^{2}$. The isotropic diffusivity $\nu$ is given by

$$
\nu(x)=e^{-5\left|x-x_{0}\right|^{2}},
$$

where $x_{0}=(1.5,-1.5)$. The diffusion is therefore concentrated in a corner on the boundary of the set $\Sigma$. The effect of this diffusion is to move mass from the interior of $\Sigma$ to the exterior, in the neighbourhood of the point $x_{0}$. Figure 4 shows the numerical solution along with its level curves at $T=2$, computed on a $50 \times 50$ space grid using $R_{0}$ reconstruction and time step $\Delta t=0.05$. Note that, despite being conceptually obtained by directional splitting, the two-dimensional scheme (21) does not suffer from anisotropies induced by grid orientation.

\section{Conclusions}

We have extended the SL approach of [9] to achieve a fully conservative, flux form discretization for linear and nonlinear parabolic problems. The resulting method is simpler to implement than the original non conservative variant, since the computation of the diffusive displacements $\delta_{i}^{ \pm}$is explicit and does not require the solution of a nonlinear equation. A consistency and stability analysis of the method has also been presented, along with a strategy to couple the FFSL discretizations of advection and diffusion terms. Various numerical simulations validate the proposed method, showing that it is equivalent in accuracy to its nonconservative variant, while allowing to maintain mass conservation at machine accuracy. The proposed method could represent an important tool to treat diffusive terms in combination with the many conservative, flux-form semi-Lagrangian schemes for advection terms proposed in the literature, with the advantage of going beyond CFL restrictions at a lower cost than implicit methods.

Future developments will focus on the application of the proposed approach to conservation laws with nonlinear parabolic terms, such as e.g. the Richards equation, and to the extension of this technique to high order discontinuous finite elements discretizations such as those proposed in [15], [35], [36]. 


\section{Flux form SL methods for parabolic problems}

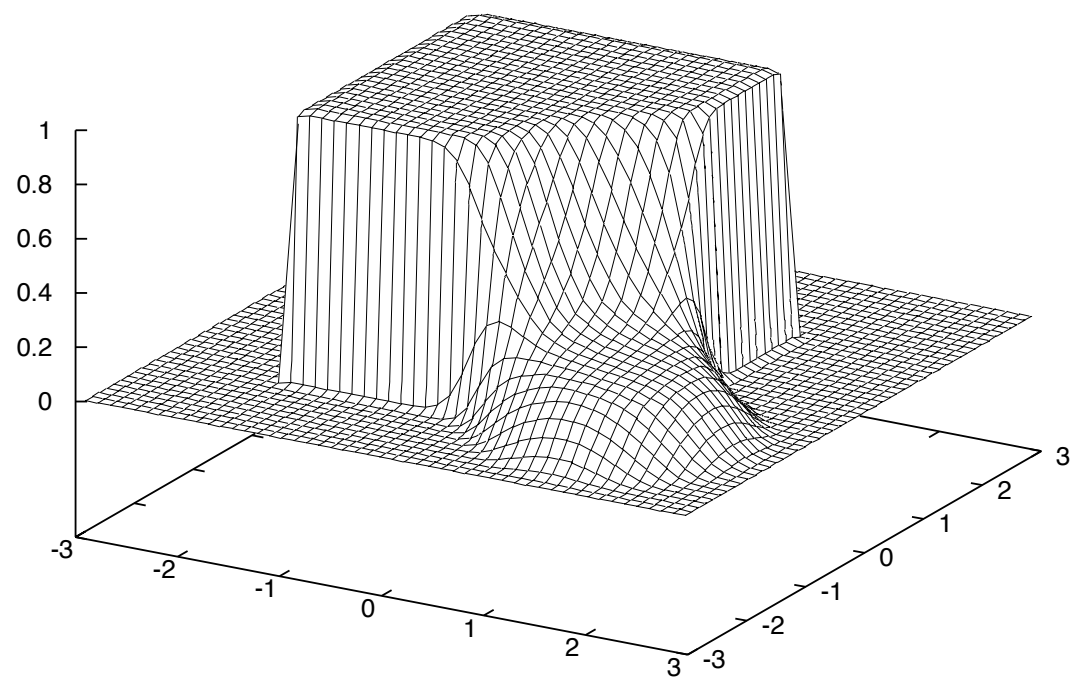

(a)

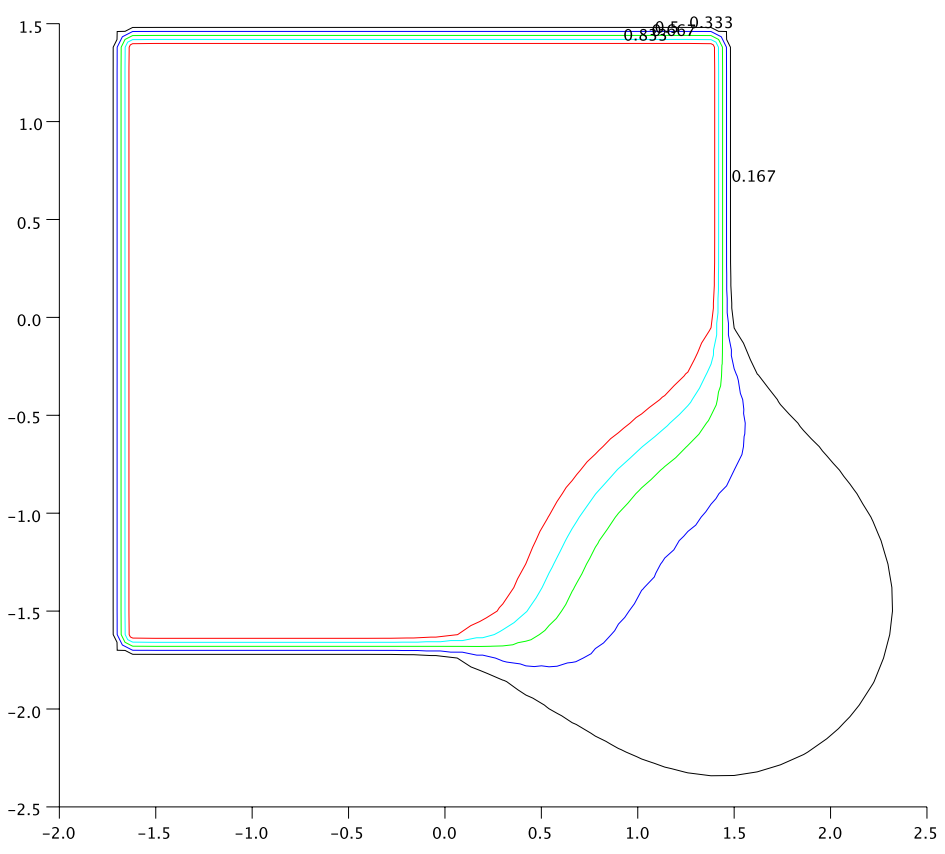

(b)

Figure 4. Variable isotropic diffusion, graph (a) and level curves (b) of the solutions. 
Bonaventura, Ferretti

\section{Acknowledgements}

This research work has been financially supported by the INDAM-GNCS project Metodi numerici semi-impliciti e semi-Lagrangiani per sistemi iperbolici di leggi di bilancio and by Politecnico di Milano and University Roma Tre. We would like to thank two anonymous reviewers for their constructive comments, that helped improving the original version of the paper, as well as Giovanni Russo for several useful conversations on the topics related to this paper.

\section{REFERENCES}

1. M. Falcone and R. Ferretti, Semi-Lagrangian Approximation Schemes for Linear and Hamilton-Jacobi Equations. SIAM, 2013.

2. M. Camilli and M. Falcone, An approximation scheme for the optimal control of diffusion processes., M2AN, vol. 29, pp. 97-122, 1995.

3. G. Milstein, The probability approach to numerical solution of nonlinear parabolic equations., Numerical Methods for Partial Differential Equations, vol. 18, pp. 490-522, 2002.

4. G. Milstein and M. Tretyakov, Numerical algorithms for semilinear parabolic equations with small parameter based on approximation of stochastic equations., Mathematics of Computation, vol. 69, pp. 237-567, 2000.

5. G. Milstein and M. Tretyakov, Numerical solution of the dirichlet problem for nonlinear parabolic equations by a probabilistic approach., IMA Journal of Numerical Analysis, vol. 21, pp. 887-917, 2001.

6. R. Ferretti, A technique for high-order treatment of diffusion terms in semi-Lagrangian schemes., Communications in Computational Physics, vol. 8, pp. 445-470, 2010.

7. J. Teixeira, Stable schemes for partial differential equations: The one-dimensional diffusion equation., Journal of Computational Physics, vol. 153, pp. 403-417, 1999.

8. L. Bonaventura, R. Redler, and R. Budich, Earth System Modelling 2: Algorithms, Code Infrastructure and Optimisation. New York: Springer Verlag, 2012.

9. L. Bonaventura and R. Ferretti, Semi-Lagrangian methods for parabolic problems in divergence form., SIAM Journal of Scientific Computing, vol. 36, pp. A2458 - A2477, 2014.

10. B. Leonard, A. Lock, and M. MacVean, Conservative explicit unrestricted-time-step multidimensional constancy-preserving advection schemes., Monthly Weather Review, vol. 124, pp. 2588-2606, November 1996.

11. S. Lin and R. B. Rood, Multidimensional flux-form semi-Lagrangian transport schemes., Monthly Weather Review, vol. 124, pp. 2046-2070, September 1996.

12. P. Frolkovic, Flux-based method of characteristics for contaminant transport in flowing groundwater., Computing and Visualization in Science, vol. 5, pp. 73-83, 2002.

13. E. S. N. Crouseilles, M. Mehrenberger, Conservative semi-Lagrangian schemes for the Vlasov equation., Journal of Computational Physics, vol. 229, pp. 1927-1953, 2010.

14. T. Phillips and A. Williams, Conservative semi-Lagrangian finite volume schemes., Numerical Methods for Partial Differential Equations, vol. 17, pp. 403-425, 2001.

15. M. Restelli, L. Bonaventura, and R. Sacco, A semi-Lagrangian Discontinuous Galerkin method for scalar advection by incompressible flows, Journal of Computational Physics, vol. 216, pp. 195-215, 2006. 


\section{Flux form SL methods for parabolic problems}

16. J. Qiu and C. Shu, Conservative high order semi-Lagrangian finite difference WENO methods for advection in incompressible flow., Journal of Computational Physics, vol. 230 , p. $863 \dddot{i} ; \frac{1}{2} 889,2011$.

17. J. Qiu and C. Shu, Positivity preserving semi-Lagrangian discontinuous Galerkin formulation: Theoretical analysis and application to the Vlasov-Poisson system., Journal of Computational Physics, vol. 230, p. 8386ï $\frac{1}{2} 8409,2011$.

18. J. Rossmanith and D. Seal, A positivity-preserving high-order semi-Lagrangian discontinuous Galerkin scheme for the Vlasovï; $\frac{1}{2}$ Poisson equations., Journal of Computational Physics, vol. 230, pp. 6203-6232, 2011.

19. J. Dukowicz, Conservative rezoning (remapping) for general quadrilateral meshes., Journal of Computational Physics, vol. 54, pp. 411-424, 1984.

20. J. Laprise and R. Plante, A class of semi-Lagrangian integrated-mass (SLIM) numerical transport algorithms., Monthly Weather Review, vol. 123, pp. 553-565, 1995.

21. L. Leslie and R. Purser, Three-dimensional mass-conserving semi-Lagrangian scheme employing foreward trajectories., Monthly Weather Review, vol. 123, pp. 2551-2566, 1995.

22. B. Machenhauer and M. Olk, The implementation of the semi-implicit scheme in cellintegrated semi-Lagrangian models., Atmosphere-Ocean, vol. XXXV, pp. 103-126, March 1997.

23. M. Rancic, An efficient, conservative, monotone remapping for semi-Lagrangian transport algorithms., Monthly Weather Review, vol. 123, pp. 1213-1217, 1995.

24. J. Dukowicz and J. Baumgardner, Incremental remapping as a transport/advection algorithm., Journal of Computational Physics, vol. 160, pp. 318-335, 2000.

25. W. Lipscomb and T. Ringler, An incremental remapping transport scheme on a spherical geodesic grid., Monthly Weather Review, vol. 133, pp. 2335-2350, 2005.

26. R. Nair and B. Machenhauer, The mass-conservative cell-integrated semi-Lagrangian advection scheme on the sphere., Monthly Weather Review, vol. 130, pp. 649-667, 2002.

27. M. Zerroukat, N. Wood, and A. Staniforth, SLICE: a semi-Lagrangian inherently conserving and efficient scheme for transport problems., Quarterly Journal of the Royal Meteorological Society, vol. 128, pp. 2801-2820, 2002.

28. M. Zerroukat, N. Wood, and A. Staniforth, SLICE-S: A semi-Lagrangian inherently conserving and efficient scheme for transport problems on the sphere., Quarterly Journal of the Royal Meteorological Society, vol. 130, pp. 2649-2664, 2004.

29. M. Zerroukat, N. Wood, and A. Staniforth, A monotonic and positive-definite filter for a semi-Lagrangian inherently conserving and efficient (SLICE) scheme., Quarterly Journal of the Royal Meteorological Society, vol. 131, pp. 2923-2936, 2005.

30. M. Zerroukat, N. Wood, A. Staniforth, A. A. White, and J. Thuburn, An inherently conserving semi-implicit semi-Lagrangian discretisation of the shallow water equation on the sphere., Quarterly Journal of the Royal Meteorological Society, vol. 135, pp. 1104-1116, 2009.

31. R. Ferretti, Stability of some generalized Godunov schemes with linear high-order reconstructions., Journal of Scientific Computing, vol. 57, pp. 213-228, 2013.

32. R. Ferretti, Equivalence of semi-Lagrangian and Lagrange-Galerkin schemes under constant advection speed., Journal of Computational Mathematics, vol. 28, pp. 461$473,2010$. 


\section{Bonaventura, Ferretti}

33. E. Carlini, M. Falcone, and R. Ferretti, A Time - Adaptive Semi-Lagrangian Approximation to Mean Curvature Motion, in Numerical mathematics and advanced applications, pp. 732-739, Springer Berlin Heidelberg, 2006.

34. G. I. Barenblatt, On self-similar motions of a compressible fluid in a porous medium, Akad. Nauk SSSR. Prikl. Mat. Meh, vol. 16, no. 6, pp. 79-6, 1952.

35. G. Tumolo and L. Bonaventura, A semi-implicit, semi-Lagrangian, DG framework for adaptive numerical weather prediction., Quarterly Journal of the Royal Meteorological Society, vol. 141, pp. 2582-2601, 2015.

36. G. Tumolo, L. Bonaventura, and M. Restelli, A semi-implicit, semi-Lagrangian, $p$-adaptive discontinuous Galerkin method for the shallow water equations., Journal of Computational Physics, vol. 232, pp. 46-67, 2013. 